EESTI NSV TEADUSTE AKADEEMIA TOIMETISED. XII KÖIDE FOOSIKA-MATEMAATIKA- JA TEHNIKATEADUSTE SEERIA. 1963, NR. 2

ИЗВЕСТИЯ АКАДЕМИИ НАУК ЭСТОНСКОН ССР. ТОМ ХІІ СЕРИЯ ФИЗИКО-МАТЕМАТИЧЕСКИХ И ТЕХНИЧЕСКИХ-НАУК. 1963, № 2

\title{
ВЛИЯНИЕ СОСТАВА ГОРЮЧЕГО СЛАНЦА-КУКЕРСИТА НА ТЕПЛОВЫЕ ПОТЕРИ С ПРОДУКТАМИ СЖИГАНИЯ
}

\section{Э. ЛАНДРА}

Состав сланца-кукерсита зависит от его месторождения, метода выемки и обогащения.

Содержание горючей части в рабочей массе сланца-кукерсита $\Gamma=$ $=16 \ldots 38 \%$, но его можно значительно повысить обогащением. При этом элементарный состав горючей массы колеблется в узких пределах $\left.{ }^{1}\right]$ и его можно рассматривать как независимого от месторождения, метода выемки и обогащения.

Влажность сланца-кукерсита в рабочей массе $W=10 \ldots 15 \%$, но при мокром обогащении она может увеличиться.

Минеральная часть сухого сланца-кукерсита в зависимости от месторождения и метода выемки $A=62 \ldots 84 \%$. Рабочая масса сланцакукерсита с содержанием минеральной части выше $80 \%$ имеет тепло-

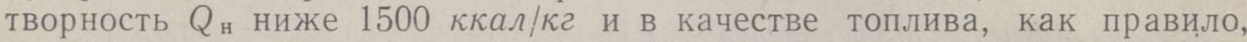
не применяется. Содержание минеральной части можно уменьшить обогащением

Знание зависимости тепловых потерь при сжигании от состава сланца-кукерсита позволяет точнее оценить метод выемки или обогащения сланца-кукерсита данного месторождения.

Для определения к.п.д. энергетических установок или тепловых потерь имеются некоторые упрощенные методы. Можно сослаться на работы проф. С. Я. Корницкого, проф. А. М. Гурвича и проф. М. Б. Равича $[2,3,4]$. Для некоторых видов топлива можно использовать построенные авторами номограммы [5,6]. Имеется сравнительно точный метод для подсчета потерь тепла с уходящими газами, который учитывает изменение влажности топлива [7].

Все названные методы имеют целью более или менее точное определение к.п.д. или потерь тепла с уходящими газами для проверки работы конкретных установок, но не являются удобными для анализа влияния изменения состава топлива на к.п.д. или на тепловые потери. Этот вопрос имеет особое значение при сжигании такого многозольного топлива, как сланец-кукерсит.

Ниже исследуется влияние состава сланца-кукерсита на теплосодержание уходящих газов $Q_{2}$ и теплосодержание золы $Q_{6}$, причем $Q_{2+6}=$ $=Q_{2}+Q_{6}$.

Чтобы выяснить влияние на потери каждой составляющей, необходимо определить теплосодержание продуктов сжигания 1 кट горючей массы, минеральной части и влаги отдельно, а при суммировании теплосодержаний умножить каждый вид на коэффициент, показывающий содержание данной составляющей в рабочем топливе. 
В общем случае теплосодержание уходящих газов и золы при сжигании горючего сланца можно выразить формулой:

$$
\begin{aligned}
Q_{2+6}= & {\left[I_{\mathrm{r}}+I_{\alpha} \cdot(\alpha-1)\right]\left(1-\frac{q_{4}}{100}\right) \frac{\Gamma}{100}+1,24 I_{\mathrm{H}_{2} \mathrm{O}} \frac{W}{100}+} \\
+ & 0,509 I_{\mathrm{RO}_{2}} \cdot a \cdot k \cdot \frac{A}{100}+I_{3 \mathrm{I}} \cdot a_{\mathrm{yн}}(1-a \cdot k) \frac{A}{100}+ \\
& +I_{3} \cdot\left(1-a_{\mathrm{yH}}\right) \cdot(1-a \cdot k) \frac{A}{100} \quad[\kappa \kappa a \Omega / \kappa 2],
\end{aligned}
$$

где $Q_{2+6}$ - теплосодержание уходящих газов и золы в ккал на $1 \kappa$ ? рабочего топлива,

$\Gamma$ - содержание горючей массы в рабочем топливе, \%,

$W$ - содержание влаги в рабочем топливе, \%,

$A$ - содержание минеральных веществ в рабочем топливе, \%,

$I_{\text {r }}$ - теплосодержание продуктов горения горючей массы топлива при температуре уходящих газов $t_{\mathrm{yx}}$ в ккал на 1 ке горючей части, с учетом теплосодержания холодного воздуха,

$I_{\alpha}$ - теплосодержание воздуха, теоретически необходимое для полного сжигания 1 кट горючей массы топлива, при температуре уходящих газов $t_{\mathrm{yx}}$ в ккал на $1 \kappa 2$ горючей части, с учетом теплосодержания холодного воздуха,

$I_{\mathrm{H}_{2} \mathrm{O}}$ - теплосодержание $1 \mathrm{H}^{3}$ водяных паров при температуре уходящих газов $t_{\mathrm{yx}}, \kappa \kappa a \Omega / \mu \mu^{3}$,

$I_{\mathrm{RO}_{2}}$ - теплосодержание $1 \mathrm{H}^{3}$ углекислого газа при температуре уходящих газов $t_{\mathrm{yx}} \kappa \kappa a \Omega / \mu \mu^{3}$,

$I_{3}$ - теплосодержание 1 к2 золы при температуре удаления золы $t_{3}=600^{\circ} \mathrm{C}, \kappa \kappa a \Omega / \kappa 2$,

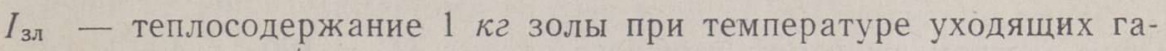
зов, ккал/кг,

$\alpha$ - коэффициент избытка воздуха,

$q_{4}$ - механический недожог, \%,

$a$ - содержание углекислого газа в минеральной части топлива,

$$
a=\frac{C O_{2}}{A}[\kappa 2 / \kappa 2]
$$

$k$ - коэффициент разложения карбонатов,

$a_{\text {ун }}-$ коэффициент уноса золы,

1,24 - объем $1 \kappa 2$ водяных паров $\mu^{3} / \kappa 2$,

0,509 - объем 1 ка углекислого газа $\mu^{3} / \kappa 2$.

Для упрощения анализа этой формулы можно принять следующие допущения.

Элементарный состав горючей массы сланца-кукерсита, в зависимости от месторождения и метода выемки, колеблется в узких пределах, и его можно считать постоянным. Теплосодержание продуктов сгорания горючей массы $I_{\text {г }}$ зависит, следовательно, только от температуры уходящих газов. 
Содержание углекислого газа в минеральной части сланца-кукерсита $a=0,23 \ldots 0,33$ и в среднем принимается $a=0,27$, Коэффициент разложения карбонатов сланца-кукерсита $k \approx 0,7$, при слоевом и $k \approx 1,0$ при пылевидном сжигании. Коэффициент уноса золы принимается $a_{\mathrm{yн}}=0$ при слоевом и $a_{\text {ун }}=0,7$ при пылевидном сжигании.

На основе этих данных по нормативному методу [8] можно определить теплосодержание продуктов сжигания $Q_{2+6}$ при данной температуре уходящих газов.

В обшем виде формула (1) следующая:

$$
Q_{2+6}=b \cdot \Gamma+m \cdot W+n \cdot A \quad[\kappa \kappa a \Omega / \kappa 2],
$$

где $b, m, n-$ коэффициенты, зависящие от температуры уходящих газов.

Температура уходящих газов при слоевом сжигании топлива редко превышает $400^{\circ} \mathrm{C}$, а при пылевидном сжигании $200^{\circ} \mathrm{C}$; нижний предел температуры уходяших газов составляет $100^{\circ} \mathrm{C}$. В этих пределах температур уходящих газов зависимости коэффициентов $b, m, n$ от $t_{\mathrm{yx}}$ можно рассматривать как приближенно линейные. Для каждого интервала температур уходящих газов можно найти соответствующую прямую, которая с достаточной точностью дает $b$ или $m$ или $n$, и определить уравнение этой прямой.

В результате расчетов при пылевидном сжигании $\left(t_{\mathrm{yx}}=100 \ldots\right.$ $\left.200^{\circ} \mathrm{C}\right)$ сланца-кукерсита теплосодержание $Q_{2+6}$ может быть выражено формулой

$$
\begin{aligned}
& Q_{2+6}=\left[\left(3,35 t_{\mathrm{yx}}-102\right)+\left(2,84 t_{\mathrm{yx}}-88\right)(\alpha-1)\right]\left(1-\frac{q_{4}}{100}\right) \frac{\Gamma}{100}+ \\
& +\left(0,455 t_{\mathrm{yx}}-0.87\right) \frac{W}{100}+\left(0,170 t_{\mathrm{yx}}+27,8\right) \frac{A}{100} \quad[\kappa \kappa a \Omega / \kappa 2]
\end{aligned}
$$

и при слоевом сжигании горючего сланца $\left(t_{\mathrm{yx}}=100 \ldots 400^{\circ}\right)-$

$$
\begin{aligned}
& Q_{2+6}=\left\{\left(3,32 t_{\mathrm{yx}}-103,8\right)[1+0,86(\alpha-1)]\right\}\left(1-\frac{q_{4}}{100}\right) \frac{\Gamma}{100}+ \\
& +\left(0,465 t_{\mathrm{yx}}-2,6\right) \frac{W}{100}+\left(0,045 t_{\mathrm{yx}}+107,8\right) \frac{A}{100} \quad[\kappa \kappa a \Omega / \kappa 2] .
\end{aligned}
$$

Погрешность результатов расчетов по формулам (3) и (4) в этих пределах $t_{\mathrm{yx}}$ по сравнению с данными, полученными по нормативному методу теплового расчета, не превышает $0,4 \%$.

Для анализа влияния состава топлива на тепловые потери с продуктами сжигания формулы (3) и (4) недостаточны, так как эти потери зависят от располагаемой теплотворной способности топлива и определяются по формуле

$$
4_{2+6}=\frac{Q_{2+6}}{Q_{p}}
$$

Для сланца-кукерсита $Q_{p}$ выражается формулой 


$$
Q_{\mathrm{p}}=Q_{\mathrm{Hr}} \cdot \frac{\Gamma}{100}-6 \mathrm{~W}-Q_{\mathrm{CO}_{2}} \cdot a \cdot k \cdot A[\kappa \kappa a \Omega / \kappa 2]
$$

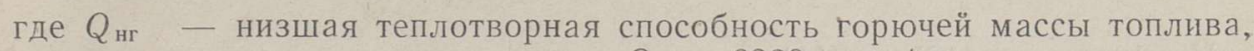
для сланца-кукерсита $Q_{\text {нг }}=8320$ ккал/кг;

$\mathrm{QCO}_{2}$ - тепло разложения карбонатов, для сланца-кукерсита $Q_{\mathrm{CO}_{2}}=9,7 \kappa \kappa a \Omega / 1 \% \quad \mathrm{CO}_{2}$.

Наряду с разложением карбонатов в минеральной части сланца кукерсита могут иметь место и другие сложные химические реакции, но суммарный тепловой эффект этих реакций в первом приближении можно ге учитывать.

Если предполагать, что $\Gamma+W+A=100$, то можно из формулы (6) элиминировать одну из составляющих топлива, например Г. После замены $Q_{\text {нг }}$, а также коэффициентов $a$ и $\kappa$ цифровыми данными формула (6) приобретает следующий вид:

$$
Q_{\mathrm{p}}=8320-89,2 \mathrm{~W}-85,82 \mathrm{~A}[\kappa \kappa a \Omega / \kappa 2]
$$

при пылевидном сжигании, а при слоевом сжигании

$$
Q_{\mathrm{p}}=8320-89,2 \mathrm{~W}-85,03 \mathrm{~A}[\kappa \kappa a \Omega / \kappa 2] .
$$

К аналогичной форме можно привести и формулу (3) или (4).

Если взять $\alpha=1, q_{4}=0$ и $t_{\mathrm{yx}}=300^{\circ} \mathrm{C}$ и учитывать условие $\Gamma+W+$ $+A=100$, то формула (4) преобразуется к следующему виду:

$$
Q_{2+6}=893-7,560 W-7,717 A[\kappa \kappa a \Omega / \kappa 2] .
$$

Таким образом, в обшем случае можно написать выражение для определения потерь с уходящими газами и золой в следующем виде:

$$
q_{2+6}=\frac{Q_{2+6}}{Q_{p}}=\frac{C-m W-n A}{D-u W-v A}
$$

При определенном значении $Q_{p}$ в общем случае $q_{2+6}$ зависит от соотношения $W$ и $A$, которые могут изменяться в некоторых пределах. Можно найти условия, при которых $q_{2+6}$ при постоянном $Q_{p}$ не зависит от соотношения $W$ и $A$.

Из зависимости

$$
Q_{p}=D-u W-v A
$$

получаем

$$
A=\frac{D-Q_{\mathrm{p}}}{v}-\frac{u}{v} W,
$$

а соответственно из

$$
Q_{2+6}=C-m W-n A
$$

получаем 


$$
A=\frac{C-Q_{2+6}}{n}-\frac{m}{n} W .
$$

Из формулы (10) и (11) получается

$$
\frac{D-Q_{\mathrm{p}}}{v}-\frac{C-Q_{2+6}}{n}=\left(\frac{u}{v}-\frac{m}{n}\right) W .
$$

Так как рассматривается случай постоянного $Q_{p}$, то левая часть уравнения (13) состоит из постоянных слагаемых. При переменном $W$ это уравнение выполняется только в случае, если

$$
\frac{u}{v}=\frac{m}{n} \text {. }
$$

При слоевом сжигании сланца-кукерсита

$$
\frac{u}{v}=\frac{89,2}{85,03}=1,049,
$$

при пылевидном сжигании

$$
\frac{u}{v}=\frac{89,2}{85,82}=1,039 .
$$

В обшем, конечно,

$$
\frac{u}{v} \neq \frac{m}{n}
$$

Для выполнения условия (14) можно коэффициент $m$ или $n$ в формуле (10) разбить на две части таким образом, что выполняется условие (13). Например, уравнение (9) можно написать в виде $\left(t_{\mathrm{yx}}=300^{\circ} \mathrm{C}\right)$

$$
\begin{aligned}
Q_{2+6} & =893-7,560 \mathrm{~W}-7,717 \mathrm{~A}= \\
& =893-7.560 \mathrm{~W}-7,207 \mathrm{~A}-0,510 \mathrm{~A}[\mathrm{\kappa \kappa a \Omega} / \kappa 2] .
\end{aligned}
$$

Формула (15) без последнего слагаемого удовлетворяет условию (14). Эту часть можно использовать для расчета условных потерь $q_{2+6}^{\prime}$, которые не зависят от соотношения $W$ и $A$, а только от температуры уходящих газов и располагаемой теплотворности сланца $Q_{\mathrm{p}}$.

Предположим первоначально, что $\alpha=1$, и определим зависимость $q_{2+6}^{\prime}$ от $Q_{\mathrm{p}}$ при различных температурах уходящих газов и различных значениях $q_{4}$. На графике с логарифмическим. масштабом эти кривые можно заменить прямыми (рис. 1). Из этого следует, что $q_{2+6}^{\prime}$ можно приближенно выразить формулой

$$
q^{\prime}{ }_{2+6}=p \cdot\left(Q_{\mathrm{p}}\right)^{t} .
$$

Угловой коэффициент, который соответствует степени $t$ в формуле (16), мало зависит от $t_{\mathrm{yx}}$ и от потерь механического недожога $q_{4}$. Величина его довольно близка к $-0,5$ (см. рис. 1). 
Коэффициент $p$, который на рис. 1 соответствует точке пересечения прямой с осью ординат, зависит от $t_{\mathrm{yx}}$, а также от $q_{4}$ (рис. 2 ).

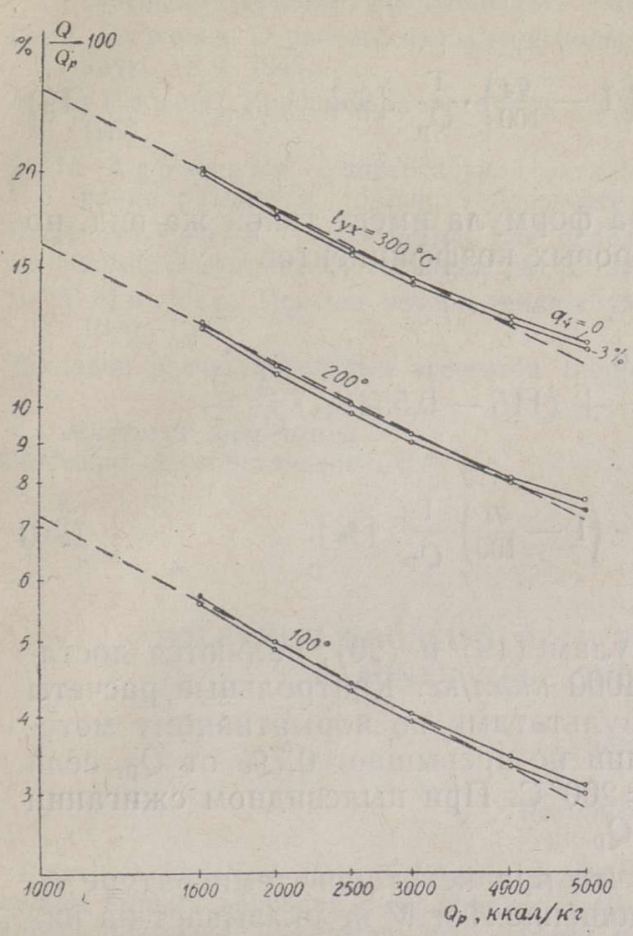

Рис. 1. $q_{2+6}=f\left(Q_{\mathrm{p}}^{\mathrm{p}}\right)$ при пылевидном сжигании сланца-кукерсита при $\alpha=1$.

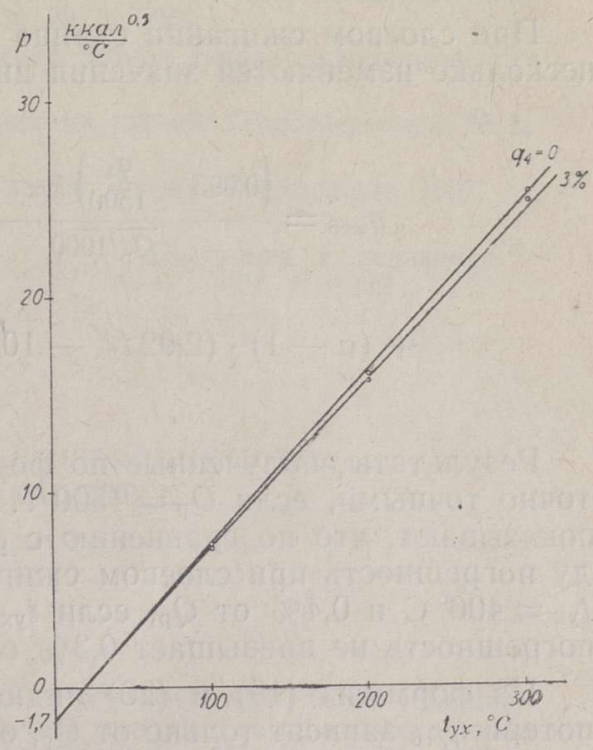

Рис. 2. $p=f\left(t_{\mathrm{yx}}\right)$ при пылевидном сжигании сланца-кукерсита (формула 17).

Эта зависимость линейна и ее можно выразить формулой

$$
p=\left(0,0905-\frac{q_{4}}{1000}\right) \cdot t_{\mathrm{yx}}-1,7
$$

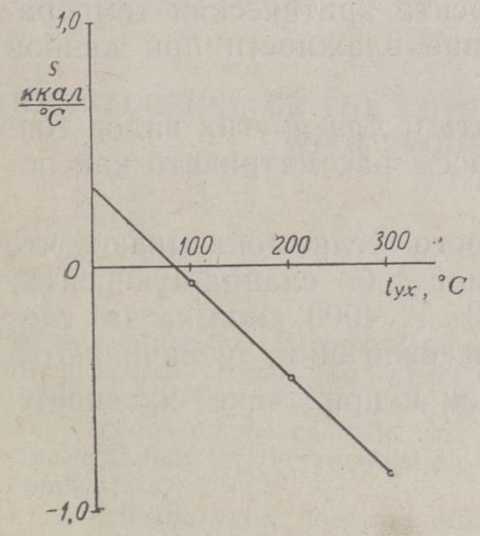

Рис. 3. $s=f\left(t_{\mathrm{yx}}\right)$ при пылевидном сжигании сланца-кукерсита (формула 18).

Чтобы получить формулу для $q_{2+6}$, необходимо учитывать последнее слагаемое в формуле типа (15). Коэффициент этого слагаемого зависит от температуры уходяших газов, что видно из формулы (3). Эта линейная зависимость представлена на рис. 3; ее можно выразить формулой

$$
s=0,32-0,0039 t_{\mathrm{yx}}
$$

Дополнительно следует учитывать влияние избытка воздуха. Для этого можно пользоваться вторым слагаемым формулы (3).

В конечном итоге потери с продуктами сжигания сланца-кукерсита при пылевидном сжигании выразятся формулой 


$$
\begin{aligned}
q_{2+6} & =\frac{\left(0,0905-\frac{q_{4}}{1000}\right) t_{\mathrm{yx}}-1,7}{\sqrt{Q_{\mathrm{p}} / 1000}}+\left(32-0,39 t_{\mathrm{yx}}\right) \frac{A}{Q_{\mathrm{p}}}+ \\
& +(\alpha-1) \cdot\left(2,84 t_{\mathrm{yx}}-88\right)\left(1-\frac{q_{4}}{100}\right) \cdot \frac{\Gamma}{Q_{\mathrm{p}}}[\%]
\end{aligned}
$$

При слоевом сжигании сланца эта формула имеет такой же вид, но несколько изменяются значения цифровых коэффициентов

$$
\begin{gathered}
q_{2+6}=\frac{\left(0,095-\frac{q_{4}}{1500}\right) t_{\mathrm{yx}}-2,9}{\sqrt{Q_{\mathrm{p}} / 1000}}+\left(115-0,554 t_{\mathrm{yx}}\right) \frac{A}{Q_{\mathrm{p}}}+ \\
+(\alpha-1) \cdot\left(2,92 t_{\mathrm{yx}}-103\right) \cdot\left(1-\frac{q_{4}}{100}\right) \frac{\Gamma}{Q_{\mathrm{p}}}[\%] .
\end{gathered}
$$

Результаты, полученные по формулам (19) и (20), являются достаточно точными, если $Q_{p}=1500 \ldots 4000$ ккал/к2. Контрольные расчеты показывают, что по сравнению с результатами по нормативному методу погрешность при слоевом сжигании не превышает $0,7 \%$ от $Q_{p}$, если $t_{\mathrm{yx}}=400^{\circ} \mathrm{C}$ и $0,4 \%$ от $Q_{\mathrm{p}}$, если $t_{\mathrm{yx}}=200^{\circ} \mathrm{C}$. При пылевидном сжигании погрешность не превышает $0,3 \%$ от $Q_{\mathrm{p}}$.

Из формулы (19) и (20) видно, что при некоторой температуре $t_{\text {ул }}$ потери $q_{2+6}$ зависят только от $Q_{\mathrm{p}}$, отношение $A$ и $W$ не оказывает на них влияния. При слоевом сжигании сланца-кукерсита, например, если $\alpha=1$, такой критической температурой является $210^{\circ} \mathrm{C}$. При увеличе нии $\alpha$ эта температура уменьшается, например, если $\alpha=2$, то она $\approx 175^{\circ} \mathrm{C}$.

Если $t_{\mathbf{y x}}$ ниже критической, то при данной теплотворности увеличение влажности за счет зольности сланца-кукерсита сопровождается уменьшением потери $q_{2+6}$. Если $t_{\mathrm{yx}}$ выше критической, то наоборот, увеличение влажности за счет зольности при данной $Q_{p}$ увеличивает $q_{2+6}$.

При пылевидном сжигании сланца-кукерсита критическая температура ниже $100^{\circ} \mathrm{C}$, таким образом, увеличение влажности при данной $Q_{\mathrm{p}}$ всегда увеличивает $q_{2+6}$.

Вышеприведенный метод можно применить и для других видов топлива, если элементарный состав горючей массы рассматривать как постоянный.

Расчеты для торфа и подмосковного бурого угля показывают, что при уменьшении низшей (или располагаемой для сланца-кукерсита)

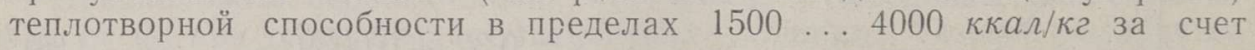
влажности или золы, потери с продуктами сжигания увеличиваются обратно пропорционально величине $\sqrt{Q_{\mathrm{p}}}$, как и при сланце-кукерсите.

\section{Л ИТЕРАТ У РА}

1. Х. Т. Р аудсепп, А. С. Фомина, Б. К. Торпан, Х. К. Норман. Техникохимическая характеристика кукерского сланца западного крыла Эстонского сланцевого бассейна. Тр. Таллинск. политехн. ин-та. Сер. А, № 57, 1954, Сб. статей по химии и технологии горючего сланца. 
2. С. Я. Корн ицки й, С. В. Т а ти щ е в, Методика нормирования расхода топлива для отопления котлов малой и средней производительности, оборудованных слоевыми топками. Госпланиздат, 1948.

3. А. М. Г у р в и ч, О расчете продуктов полного сгорания. Изв. АН СССР, Отд. техн. наук, № 9, 1945.

4. М. Б. Р а в и ч, Упрощенная методика теплотехнических расчетов. Изд. АН СССР, 1958.

5. А. Н. А дри анов, Универсальная энтальнийная диаграмма для продуктов сгорания различных топлив. Теплоэнергетика, № 3, 1959.

6. Б. И. Логинов, Номограммы для определения потери тепла с уходящими газами. Электрические станции № 8, 1960.

7. Я. Л. Пеккер, Подсчет потери тепла с уходящими газами. Теплоэнергетика № 8, 1960.

8. Тепловой расчет котельных агрегатов (нормативный метод). Энергоиздат, 1957.
Институт энергетики
Академии наук Эстонской ССР
Поступила в редакцию
19. II 1962

\title{
PŌLEVKIVI KOOSTISE MÕJU PŌLEMISPRODUKTIDEGA KAASNEVATELE SOOJAKADUDELE
}

\author{
E. Landra
}

\section{Resümee}

Pōlevkivi koostise mõju tundmine soojakadudele põlevkivi pōletamisel võimaldab täpsemalt hinnata põlevkivi kaevandamise ja rikastamise meetodeid. Ligikaudsed valemid põlemisproduktidega kaasnevate soojakadude $q_{2+6}$ arvutamiseks pōlevkivi kütteväärtuse ja koostise järgi esitatakse tolmpōlemise jaoks valemis (19) ja kihispōlemise jaoks valemis (20).

Valemite kasutamisel esinev viga ei ületa $0,4 \%$ kütteväärtusest, kui viimane on $1500 \ldots 4000 \mathrm{kcal} / \mathrm{kg}$ piirides. Sellise kütteväärtuse puhul on kaod $q_{2+6}$ esimeses lähenduses pöördvōrdelised ruutjuurega kütteväärtusest.
Eesti NSV Teaduste Akadeeniia
Energeetika Instituut
Saabus toimetusse
19. II 1962

\section{THE INFLUENCE OF THE COMPOSITION OF OIL SHALE ON HEAT LOSS WITH PRODUCTS OF COMBUSTION}

\author{
E. Landra
}

\section{Summary}

For estimating the methods of oil shale mining and dressing, it is necessary to know the influence of the composition of oil shale on heat loss by combustion. The author presents approximate formulas for heat loss with products of oil shale. The heat loss in these formulas depends on the calorific value and composition of oil shale. Formula (19) presents the heat loss for the combustion of pulverized oil shale, formula (20) - for layerwise combustion.

If the calorific value does not diminish below $1500 \mathrm{kcal} / \mathrm{kg}$, or exceed $4000 \mathrm{kcal} / \mathrm{kg}$, the error in these formulas is less than 0.4 per cent of the calorific value. With such calorific value, the mentioned heat loss is approximately inversely proportional to the square root of calorific value. 